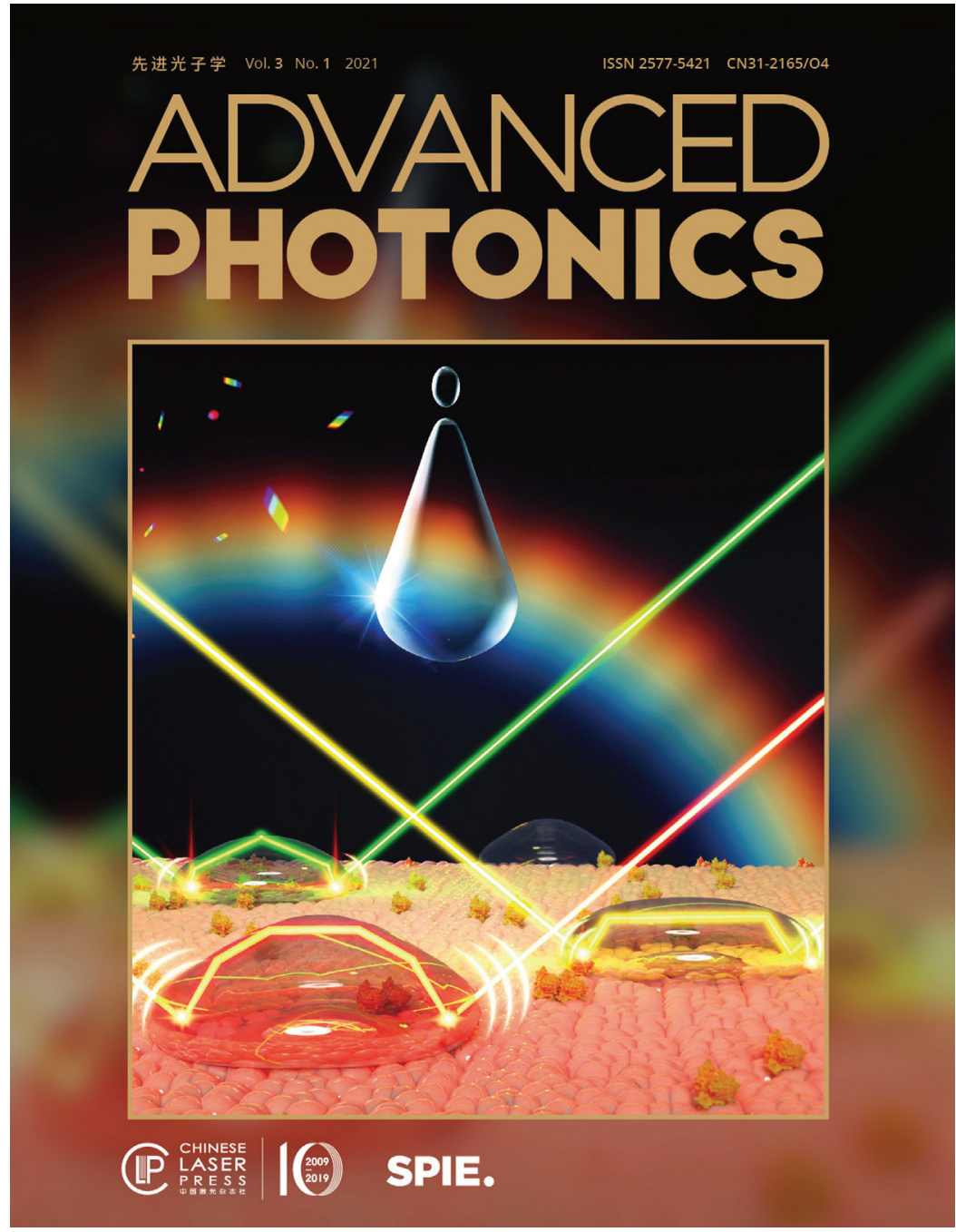

\title{
About the cover: Advanced Photonics Volume 3, Issue 1
}

The image on the cover for Advanced Photonics Volume 3 Issue 1 illustrates the concept of a new lasing mechanism discovered in water droplets. The image shows that tiny molecular forces at the droplet-air interface can dramatically change laser resonating geometry, shape lasing pathways, and modulate laser output emission wavelengths and modes. This novel concept provides a simple yet highly versatile method to manipulate laser emissions, as well as valuable insight into how molecules interact with and modulate laser light. The original research, presented in the article "Lasing action in microdroplets modulated by interfacial molecular forces" by Zhen Qiao et al., lays a foundation for the development of tunable photonic devices at the molecular level. 